\title{
Highway Middle Pile Coordinate Automatic Calculation based on Combine of Excel and Excel VBA Program
}

\author{
Guo-wen CHE \\ Baicheng Normal University, 137000, China \\ cheguowen@163.com
}

keywords: Excel software, Excel VBA program, Highway Horizontal Alignment, circular curve, transition curve, pile point coordinate

\begin{abstract}
In the highway design and construction, it is common to calculate the middle pile point coordinates. Application the data calculation, data processing function and function library of Excel software establish worksheet calculation model of route transverse. Then using Excel VBA program achieves automatic calculation of road middle pile point coordinates. Results show, this fast and accurate calculation method has strong practicality and generality and can be used as a reference for the highway construction personnel.
\end{abstract}

\section{Introduction}

In the construction of highway, Global Positioning System (GPS), Total Station have been widely used which are essential especially during the construction of high-grade highway. Regardless of what the method might be, the middle pile points coordinates are need when put in the line. Although various kinds of design software can provide these data, but the use of these software are limited to the design institutes. Most of middle state points coordinates can only be manual calculated for the engineering and technical personnel not working in the design institute. The number of middle pile points are hundreds, thousands or more in a route, so the calculation is a great work and long period by hand. Besides the huge workload and complex calculation procedures, it is easy to appear mistake and the calculation precision is difficult to guarantee. It can avoid the defects of manual calculation if the work accomplished by the software Excel and VBA. Excel software itself has a powerful calculation function but cannot be complex logic judgment and duplicated data processing. If combining Excel software and VBA program, the fast and high precision automatic calculation can be realized for the highway middle pile points coordinates.

\section{Coordinate Azimuth $A$, Length $S$, Rotating $\alpha$ Calculation of Traverse}

It is need base data during highway middle pile points coordinates calculation, such as route intersection points coordinates, intersection points distance, computation azimuth, corners, horizontal curve elements and so on. Established route intersection coordinates in the route design are known data. Route middle pile points range and coordinates are calculated by Excel and VBA program, and most of the rest basic data are calculated by Excel software to complete.

As shown in Figure1, coordinate $X_{\mathrm{JD}}$ and $Y_{\mathrm{JD}}$ of Intersection $J D$ are known and the coordinate azimuth $A$, distance $S$ between the intersection of routed transverse can be calculated by the following formula.

Azimuth: $A_{i-1, i}=\operatorname{tg}^{-1} \frac{Y_{i}-Y_{i-1}}{X_{i}-X_{i-1}}$

Intersection Distance: $S=\sqrt{\left(X_{i}-X_{i-1}\right)^{2}+\left(Y_{i}-Y_{i-1}\right)^{2}}$

Where, $X_{\mathrm{i}-1}$ and $Y_{\mathrm{i}-1}$ is coordinate of point $i-1 ; X_{\mathrm{i}}$ and $Y_{\mathrm{i}}$ is coordinate of point $i$. 


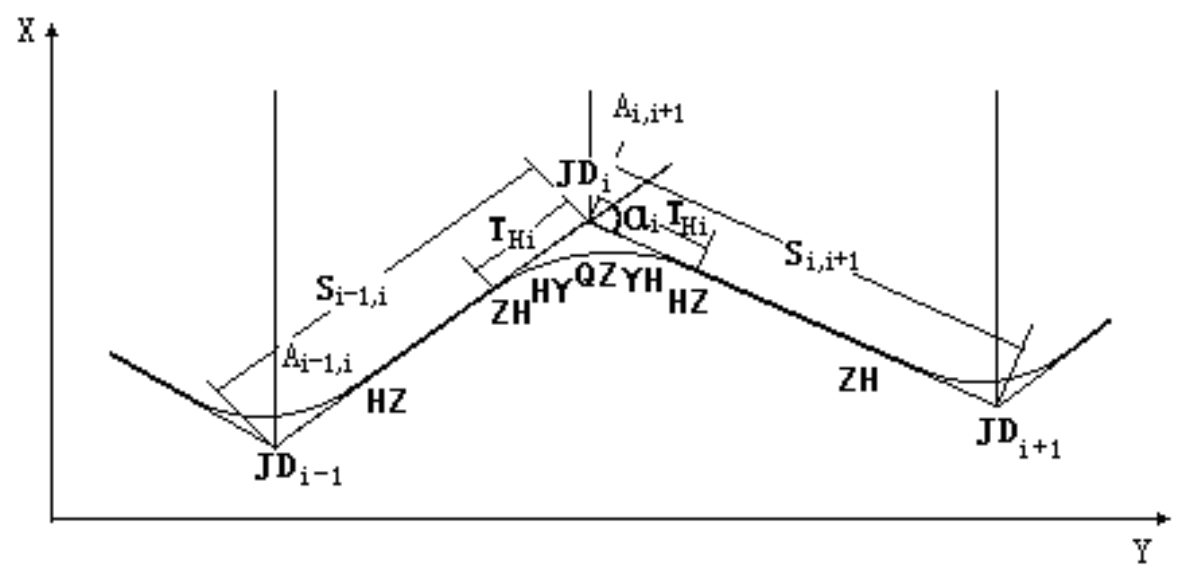

Fig.1. Middle pile coordinate calculation

\section{Middle Pile Coordinate Calculation}

Middle Pile Coordinate between HZ Point (Including Route Starting Point) and HZ Point. As shown in Figure 1, for straight line, the coordinates of pile points can be calculated as follows.

$$
\begin{aligned}
& X_{i}=X_{H Z i-1}-D_{i} \cos A_{i-1, i} \\
& Y_{i}=Y_{H Z i-1}-D_{i} \sin A_{i-1, i}
\end{aligned}
$$

Where, $A_{\mathrm{i}-1, \mathrm{I}}$ is coordinate azimuth of route transverse $J D_{\mathrm{i}-1}-J D_{\mathrm{i}}, D_{\mathrm{i}}$ is distance between pile point and point $H Z_{\mathrm{i}-1}$ which is difference between pile point mileage and point $H Z_{\mathrm{i}-1}$ mileage, $X_{\mathrm{HZi}}$ ${ }_{-1}$ and $Y_{\mathrm{HZi}-1}$ are coordinates of point $H Z_{\mathrm{i}-1}$ which can be calculated as follows.

$$
\begin{aligned}
& X_{H Z i-1}=X_{J D i-1}-T_{H i-1} \cos A_{i-1, i} \\
& Y_{H Z i-1}=Y_{J D i-1}-T_{H i-1} \sin A_{i-1, i}
\end{aligned}
$$

Where, $X_{\mathrm{JDi}-1}$ and $Y_{\mathrm{JDi}-1}$ is coordinate of intersection point $J D_{\mathrm{i}-1}, T_{\mathrm{Hi}-1}$ is tangent length, $\mathrm{ZH}$ is point of end line. Beside the formula (3) can follow, it can also be calculated as follows.

$$
\begin{aligned}
& X_{H Z i}=X_{J D i-1}+\left(S_{i-1, i}-T_{H i}\right) \cos A_{i-1, i} \\
& Y_{H Z i}=Y_{J D i-1}+\left(S_{i-1, i}-T_{H i} \sin A_{i-1, i}\right.
\end{aligned}
$$

Where, $S_{\mathrm{i}-1, \mathrm{i}}$ is side length of tangent $J D_{\mathrm{i}-1}-J D_{\mathrm{i}}$.

Pile Coordinate from Point ZH to Point YH. This segment including the first easement curve and circular curve, the tangent offset coordinate $\mathrm{x}$ and $\mathrm{y}$ of pile point can first be calculated.

Arbitrary Point on the Easement Curve. The curve equation is as follows.

$$
\left\{\begin{array}{l}
x=l-\frac{l^{5}}{40 R^{2} l_{s}^{2}} \\
y=\frac{l^{3}}{6 R l_{s}}
\end{array}\right.
$$

Where, $l$ is curve length from pile point to easement curve starting point $Z H, R$ is circular curve radius, $l_{\mathrm{s}}$ is length of easement curve.

Pile Point on the Curve. The coordinates equation of pile point on the curve is as follows.

$$
\left\{\begin{array}{l}
x=R \sin \phi+q \\
y=R(1-\cos \phi)+p
\end{array}\right.
$$

Where, $\varphi=\beta_{0}+\frac{l}{R} \frac{180^{\circ}}{\pi}, l$ is curve length form pile point to $H Y$ which is only the length of curve part. It can convert to measure coordinate $X$ and $Y$ through the coordinate transformation. The coordinate transformation as follows.

$$
\left[\begin{array}{l}
X_{i} \\
Y_{i}
\end{array}\right]=\left[\begin{array}{l}
X_{Z H i} \\
Y_{Z H i}
\end{array}\right]+\left[\begin{array}{l}
\cos A_{i-1, i}-\sin A_{i-1, i} \\
\sin A_{i-1, i}+\cos A_{i-1, i}
\end{array}\right]\left[\begin{array}{l}
x_{i} \\
y_{i}
\end{array}\right]
$$


Using formula (8) to calculate, it should substitute $y_{\mathrm{i}}=-y_{\mathrm{i}}$, when the curve turn left.

Pile Point Coordinate from Point YH to Point HZ. This segment is the second easement curve, the tangent offset coordinate can also be calculated as formula (7) and convert to measure coordinate as follows.

$$
\left[\begin{array}{l}
X_{i} \\
Y_{i}
\end{array}\right]=\left[\begin{array}{l}
X_{Z H i} \\
Y_{Z H i}
\end{array}\right]-\left[\begin{array}{l}
\cos A_{i, i+1}+\sin A_{i, i+1} \\
\sin A_{i, i+1}-\cos A_{i, i+1}
\end{array}\right]\left[\begin{array}{l}
x_{i} \\
y_{i}
\end{array}\right]
$$

It should substitute $\mathrm{y}_{\mathrm{i}}=-\mathrm{y}_{\mathrm{i}}$, when the curve turn right.

\section{Build Foundation Data Calculation Model}

Coordinate Azimuth $A$, Length $S$, Rotating a Calculating Model. Establish the coordinate azimuth $A$, length $S$, rotating ain worksheet sheet1 including coordinate increment, coordinate incremental correction, corrected increment, coordinate azimuth, rotating and so on. At the same time increase the auxiliary calculation for checking. It is relatively simple calculation that just put the formula into the corresponding cell, estimate the coordinate azimuth according the quadrant location when coordinate azimuth calculation and judge routes turning according coordinate azimuth.

It should make full use of fill handle to automatic fill data in the calculation processing and the calculation results are shown in Figure 2.

\begin{tabular}{|c|c|c|c|c|c|c|c|c|c|c|c|c|c|c|c|c|}
\hline \multirow{3}{*}{$\begin{array}{l}1 \\
2\end{array}$} & A & B & C & D & $\mathrm{E}$ & $\mathrm{F}$ & G & $\mathrm{H}$ & I & $\mathrm{J}$ & $\mathrm{K}$ & L & $M$ & $\mathrm{~N}$ & 0 & $\bar{\Delta}$ \\
\hline & \multirow[t]{2}{*}{ Intersection } & \multicolumn{2}{|c|}{$\begin{array}{l}\text { Intersection } \\
\text { coordinates }\end{array}$} & \multirow[t]{2}{*}{$\begin{array}{l}\text { Intersection } \\
\text { distance } \\
\text { (m) }\end{array}$} & \multicolumn{2}{|c|}{$\begin{array}{l}\text { Coordinate } \\
\text { increment } \\
\text { (m) }\end{array}$} & \multicolumn{2}{|c|}{$\begin{array}{l}\text { Incremental } \\
\text { correction } \\
(\mathrm{m})\end{array}$} & \multicolumn{2}{|c|}{$\begin{array}{l}\text { Corrected increment } \\
\text { (m) }\end{array}$} & \multicolumn{2}{|c|}{ Coordinate azimuth } & \multirow[t]{2}{*}{ Tuming } & \multicolumn{2}{|c|}{ Rotation Angle } & \\
\hline & & $\mathrm{N}(\mathrm{X})$ & $E(Y)$ & & $\Delta \mathrm{x}$ & $\Delta y$ & $\Delta \mathrm{x}$ & $\Delta \mathrm{y}$ & $\Delta \mathrm{x}$ & $\Delta \mathrm{y}$ & Radians & $\cdots$ & & Radians & $\cdots "$ & \\
\hline 3 & Origin & 3000.00 & 3000.00 & & & & & & & & 1.310 & $75^{\circ} 04^{\prime} \quad 06.89^{\prime \prime}$ & & & & \\
\hline 4 & JD1 & 3080.00 & 3300.00 & 310.483 & 80.00 & 300.00 & 0.00 & 0.00 & 80.00 & 300.00 & 1. 768 & $101^{\circ} 18^{\prime} 35.75^{\prime \prime}$ & Right-Turn & 0.458 & $26^{\circ} 14^{\prime} 28.85^{\prime \prime}$ & \\
\hline 5 & J12 & 3020.00 & 3600.00 & 305.941 & -60.00 & 300.00 & 0.00 & 0.00 & -60.00 & 300.00 & 1.197 & $68^{\circ} 36^{\prime} 00.68^{\prime \prime}$ & $l_{\text {eft }}-t$ urn & 0.571 & $32^{\circ} 42^{\prime} 35.07^{\prime \prime}$ & \\
\hline 6 & J13 & 3165.00 & 3970.00 & 397.398 & 145.00 & 370.00 & 0.00 & 0.00 & 145.00 & 370.00 & 2. 182 & $124^{\circ} 59^{\prime} 31.27^{\prime \prime}$ & Right-Turn & 0.984 & $56^{\circ} 23^{\prime} 30.58^{\prime \prime}$ & \\
\hline 7 & $\mathrm{~J} 44$ & 3025.00 & 4170.00 & 244.131 & -140.00 & 200.00 & 0.00 & 0.00 & -140.00 & 200.00 & 1.551 & $88^{\circ} 51^{\prime} \quad 15.25^{\prime \prime}$ & left-turn & 0.631 & $36^{\circ} 08^{\prime} 16.01^{\prime \prime}$ & \\
\hline 8 & J15 & 3030.00 & 4420.00 & 250.050 & 5.00 & 250.00 & 0.00 & 0.00 & 5. 00 & 250.00 & 2. 596 & $148^{\circ} 42^{\prime} 47.77^{\prime \prime}$ & Right-Turn & 1.045 & $59^{\circ} 51^{\prime} 32.52^{\prime \prime}$ & \\
\hline 9 & JD6 & 2770.00 & 4578.00 & 304.243 & -260.00 & 158.00 & 0.00 & 0.00 & -260.00 & 158.00 & 2. 361 & $135^{\circ} 15^{\prime} 41.84^{\prime \prime}$ & left-turn & 0.235 & $13^{\circ} 27^{\prime} 05.93^{\prime \prime}$ & \\
\hline 10 & JD7 & 2440.00 & 4905.00 & 464.574 & -330.00 & 327.00 & 0.00 & 0.00 & -330.00 & 327.00 & 1.510 & $86^{\circ} 30^{\prime} \quad 19.29^{\prime \prime}$ & left-turn & 0.851 & $48^{\circ} 45^{\prime} 22.54^{\prime \prime}$ & \\
\hline 11 & J18 & 2480.00 & 5560.00 & 656.220 & 40.00 & 655.00 & 0.00 & 0.00 & 40.00 & 655.00 & 1. 993 & $114^{\circ} 11^{\prime} 14.47^{\prime \prime}$ & Right-Turn & 0.483 & $27^{\circ} 40^{\prime} 55.17^{\prime \prime}$ & \\
\hline 12 & JD9 & 2215.00 & 6150.00 & 646.780 & -265.00 & 590.00 & 0.00 & 0.00 & -265.00 & 590.00 & 1. 209 & $69^{\circ} 15^{\prime} 14.11^{\prime \prime}$ & left-turn & 0.784 & $44^{\circ} 56^{\prime} 00.36^{\prime \prime}$ & \\
\hline 13 & JD10 & 2340.00 & 6480.00 & 352.881 & 125.00 & 330.00 & 0.00 & 0.00 & 125.00 & 330.00 & 2. 119 & $121^{\circ} \quad 25^{\prime} \quad 46.43^{\prime \prime}$ & Right-Turn & 0.911 & $52^{\circ} 10^{\prime} 32.32^{\prime \prime}$ & \\
\hline 14 & $\mathrm{~J} D 11$ & 2120.00 & 6840.00 & 421.900 & -220.00 & 360.00 & 0.00 & 0.00 & -220.00 & 360.00 & 1.581 & $90^{\circ} 33^{\prime} 42.13^{\prime \prime}$ & left-turn & 0.539 & $30^{\circ} 52^{\prime} \quad 04.29^{\prime \prime}$ & \\
\hline 15 & JD12 & 2115.00 & 7350.00 & 510.025 & -5.00 & 510.00 & 0.00 & 0.00 & -5.00 & 510.00 & 1.943 & $111^{\circ} 21^{\prime} 03.52^{\prime \prime}$ & Right-Turn & 0.363 & $20^{\circ} 47^{\prime} 21.38^{\prime \prime}$ & \\
\hline 16 & End Point & 1900.00 & 7900.00 & 590.529 & -215.00 & 550.00 & 0.00 & 0.00 & -215.00 & 550.00 & & & & & & - \\
\hline \multicolumn{17}{|l|}{17} \\
\hline 18 & $\Sigma$ & & & 5455 & -1100 & 4900 & 0 & 0 & -1100 & 4900 & & & & & & \\
\hline 19 & \multirow{4}{*}{$\begin{array}{l}\text { Auxiliary } \\
\text { calculation }\end{array}$} & $\sum \beta=$ & 2161 & $f_{f \text { 步 }}=$ & 0.000 & $\sum \Delta \mathrm{x}=$ & -1100 & $\sum \Delta_{y}=$ & 4900 & & & & & & & \\
\hline 20 & & $f_{p}=$ & 0.000 & allow & 0.000 & $f_{x}=$ & 0.000 & $f_{y}=$ & 0.000 & & & & & & & \\
\hline 21 & & $f_{\text {pallow }}=$ & 0.038 & & & $f_{\mathrm{D}}=$ & 0.000 & & & & & & & & & \\
\hline 22 & & $\Sigma \mathrm{D}=$ & 5455 & & & $\mathrm{~K}=$ & 0.000 & allow & $\mathrm{K}_{\text {anlow }}$ & 0.001 & & & & & & \\
\hline
\end{tabular}

Fig.2. The coordinate azimuth $A$ and length $S$ and rotating $\alpha$ calculating model

Horizontal Curve Elements and Main Point Mileage Calculation Model. Establish the horizontal curve elements and main point mileage calculation model in worksheet sheet 2 as show in Figure 3. Circular curve radius and easement curve length are known data or can adjust according need. The rest data only need to fill in the corresponding cell based on calculation formula and then make full use of the fill handle to populate the data automatically.

\section{Excel VBA Program Design}

Excel software itself proved to be more powerful calculation function and can complete middle pile coordinate calculation of a single horizontal curve. But there are large numbers of horizontal curve on a route, so Excel software itself cannot be logical judgement and duplicate data processing. It must use Excel VBA program to solve that problem and achieving the automatic computing of pile point mileage and pile point coordinates.

Pile Point Mileage Calculation Program Design. It must calculate the pile point mileage 
before the pile point coordinate calculation, meanwhile ensure the pile point mileage correspondence with pile coordinates. Due to the different degree of route technology level and complex terrain, the adopted pile spacing is not the same. As is well-known, modern roads are generally composed of three kinds of linear, straight line, circular curve and easement curve. Usually straight line space slightly large, pile points of curve segment will be encrypted. In order increase the versatility of program, read pile distance from worksheet Sheet2 and the next sections will describe each of program.

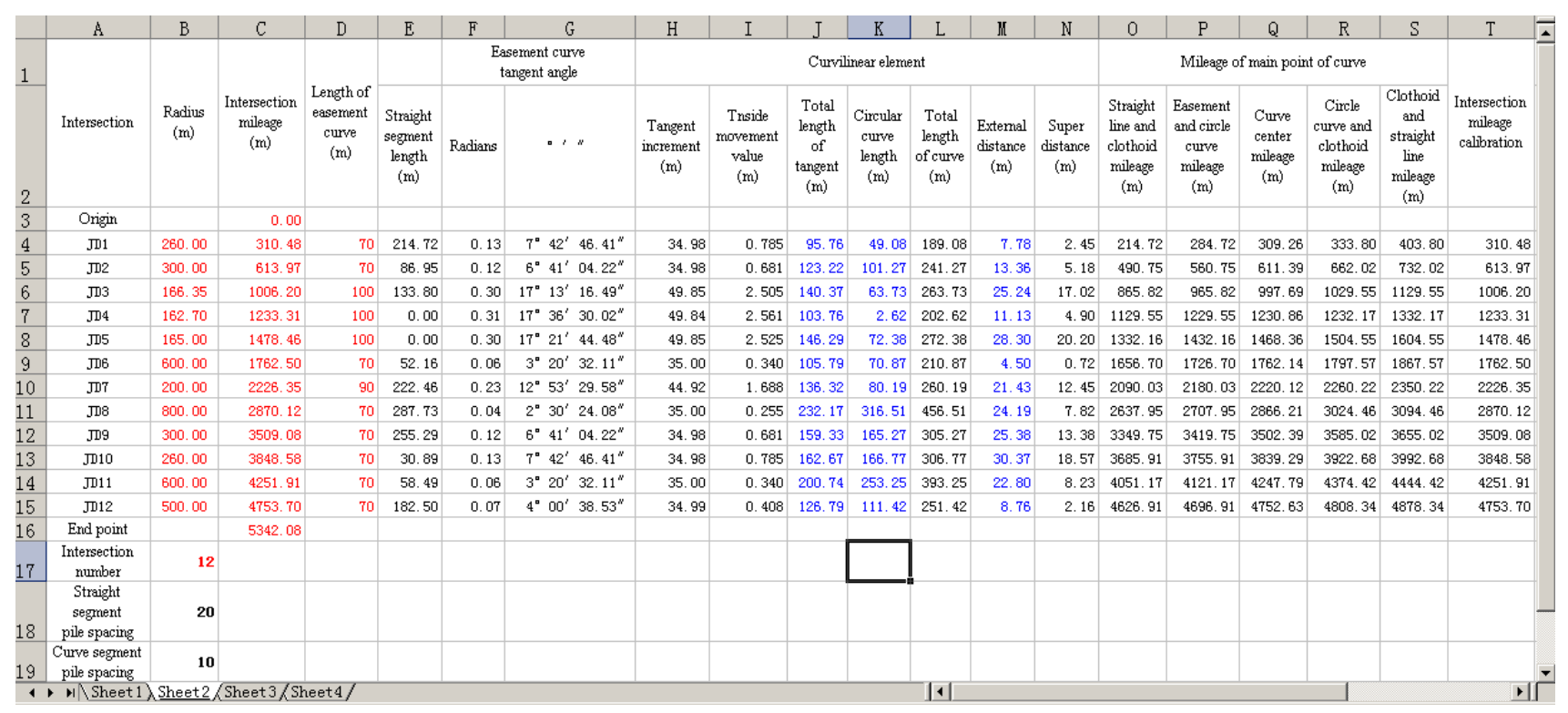

Fig.3. The horizontal curve elements and main point mileage calculation model

Read Basic Data. First define each required variable, then read the required data from Sheet2 which including them number of intersection point, starting point mileage, ending point mileage, straight-easement point mileage, easement-circular point mileage, middle curve point mileage, circular-easement point mileage, easement-straight point mileage. Procedure is as follows.

Dim j, jds, m ,zj1,zj2 As Integer

Dim qdlc, zdlc, lc As Double

Dim zh(12), hy(12), qz(12), yh(12), hz(12)

As Double

With Sheets("Sheet2")

With Sheets("Sheet2")

zj1=.Cells $(17,2)$

zj2=.Cells $(17,2)$

$\mathrm{j}=1$

qdlc $=$.Cells $(3,3)$

zdlc $=$.Cells $(16,3)$

Do While $\mathrm{j}<=$ jds

jds $=$.Cells $(17,2)$

zh(j) $=$.Cells $(j+3,15)$

hy $(j)=$.Cells $(j+3,16)$

$q z(j)=$.Cells $(j+3,17)$

$y h(j)=$. Cells $(j+3,18)$

$h z(j)=. \operatorname{Cells}(j+3,19)$

$\mathrm{j}=\mathrm{j}+1$

Loop

End With

End With

Pile Point Mileage Calculation Program Design. The constitute characteristics of route transverse are as follows, the reversed cyclic of Segment of point $H Z$ (including starting point) to point $P Z$ point, Segment of point $H Y$ to point $Y H$ point, Segment of point $Y H$ to point $H Z$ point constitute the whole road transverse. Segment of Point HZ (including route starting point) to point $\mathrm{HZ}$ is straight line and generally set the pile points by whole number method. Segment of point $\mathrm{ZH}$ to point $H Y$ and point $Y H$ to point $H Z$ are easement curve and set the pile points by whole number method or whole spacing method. It is generally adopted whole number method to set pile points for the segment of point $H Y$ to points $Y H$. In addition, the main pile point on the curve also is the control point. Program design should fully consider the characteristics of each segment. Due to the limitation, this paper give part of program source code as follows.

Middle pile points mileage calculate program between Point $H Z$ (including starting point) and point $H Z$ as follow.

Do While lc < zh(j)

If lc + zj1 < zh(j) Then 


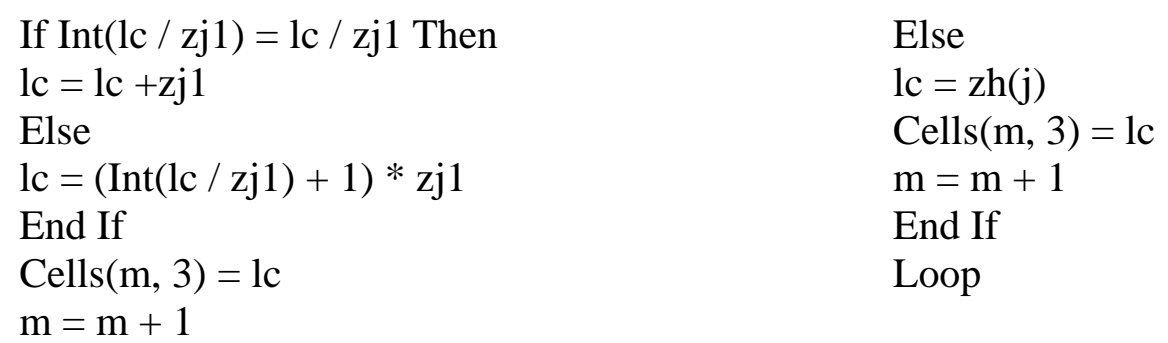

Middle pile points mileage calculate program between Point $\mathrm{ZH}$ and point $\mathrm{YH}$ as follow.

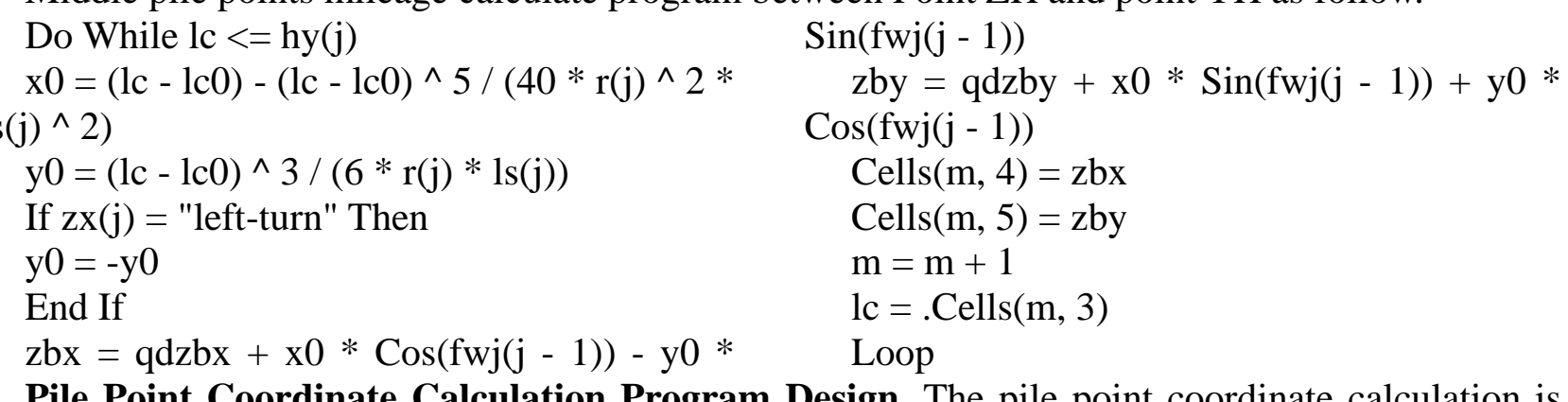

Pile Point Coordinate Calculation Program Design. The pile point coordinate calculation is similar to the pile point mileage calculation, and the base data need read from worksheet Sheet1 and Sheet2. Based on the calculation formula, beside the straight line before ending pint it can be divided into segment of point $H Z$ (including starting point) to point $H Z$, segment of point $Z H$ to point $Y H$, segment of point $Y H$ to point $H Z$.

Read basic data as follows.

Dim j, jds, m As Integer

Dim lc, qdzbx, qdzby, zbx, zby, lc0, x0, y0 As Double

Dim zh(15), hy(15), qz(15), yh(15), hz(15), fwj(15), qxzl(15) As Double

Dim r(15), ls(15), jdzbx(15), jdzby(15), qxj(15), qxc(15), nyz(15) As Double

Dim zx(15) As Variant

With Sheets("Sheet2")

jds $=$.Cells $(17,2)$

$\mathrm{j}=1$

Do While $\mathrm{j}<=\mathrm{jds}$

zh $(j)=$. Cells $(j+3,15)$

hy $(j)=$. Cells $(j+3,16)$

$\mathrm{qz}(\mathrm{j})=$. Cells $(\mathrm{j}+3,17)$

$\operatorname{yh}(j)=$. Cells $(j+3,18)$

$\mathrm{hz}(\mathrm{j})=$. Cells $(\mathrm{j}+3,19)$

$r(j)=$. Cells $(j+3,2)$

$\operatorname{ls}(\mathrm{j})=$. Cells $(\mathrm{j}+3,4)$

$q x j(j)=$. Cells $(j+3,6)$

qxzl $(j)=$. Cells $(j+3,8)$

Middle pile points coordinate calculate program between Point $Y H$ and point $H Z$ as follow.

Do While lc $<=\mathrm{hz}(\mathrm{j})$

$\mathrm{x} 0=(\mathrm{hz}(\mathrm{j})-\mathrm{lc})-(\mathrm{hz}(\mathrm{j})-\mathrm{lc}) \wedge 5 /(40 * \mathrm{r}(\mathrm{j}) \wedge 2$

$* \operatorname{ls}(\mathrm{j}) \wedge 2)$

$\mathrm{y} 0=(\mathrm{hz}(\mathrm{j})-\mathrm{lc}) \wedge 3 /(6 * \mathrm{r}(\mathrm{j}) * \mathrm{ls}(\mathrm{j}))$

If $\mathrm{zx}(\mathrm{j})=$ "Right-Turn" Then

$\mathrm{y} 0=-\mathrm{y} 0$

End If

$\mathrm{zbx}=\mathrm{qdzbx}-\mathrm{x} 0 * \operatorname{Cos}(\mathrm{fwj}(\mathrm{j}))+\mathrm{y} 0 *$

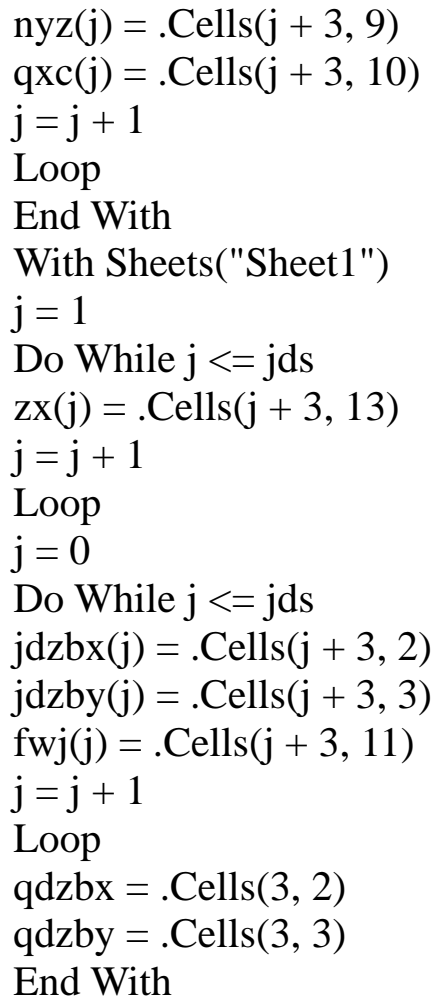

$\operatorname{Sin}(\mathrm{fwj}(\mathrm{j}))$

zby $=$ qdzby $-\mathrm{x} 0 * \operatorname{Sin}(\mathrm{fwj}(\mathrm{j}))-\mathrm{y} 0 *$

Cos(fwj(j))

Cells $(m, 4)=z b x$

Cells $(m, 5)=$ zby

$\mathrm{m}=\mathrm{m}+1$

$\mathrm{lc}=$.Cells $(\mathrm{m}, 3)$

Loop 


\section{Pile Point Mileage and Coordinate Automatic Calculation}

Within Excel, right click on menu bar above the blanks and will pop-up drop-down menu, then select "Visual Basic". Called Visual Basic menu bar displays as shown in Figure 4. Then click control toolbox shown in Figure 5 and choose command button again to insert command button which mainly used for running program when mouse click. Right click command button $\rightarrow$ command button $\rightarrow$ object $\rightarrow$ editor and enter the button name. This example adds "pile mileage calculation" and "pile coordinates calculation" two buttons. Double click the two buttons respectively and enter code editor area to insert corresponding program code. After the completion of the program editor, click the "exit design patterns" to return Excel spreadsheet and then click the button to correspond calculation. The calculation results are shown in Figure. 6.

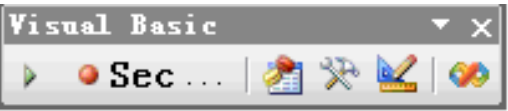

Fig.4. Visual Basic menu

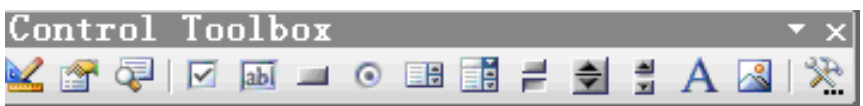

Fig.5. Control Toolbox

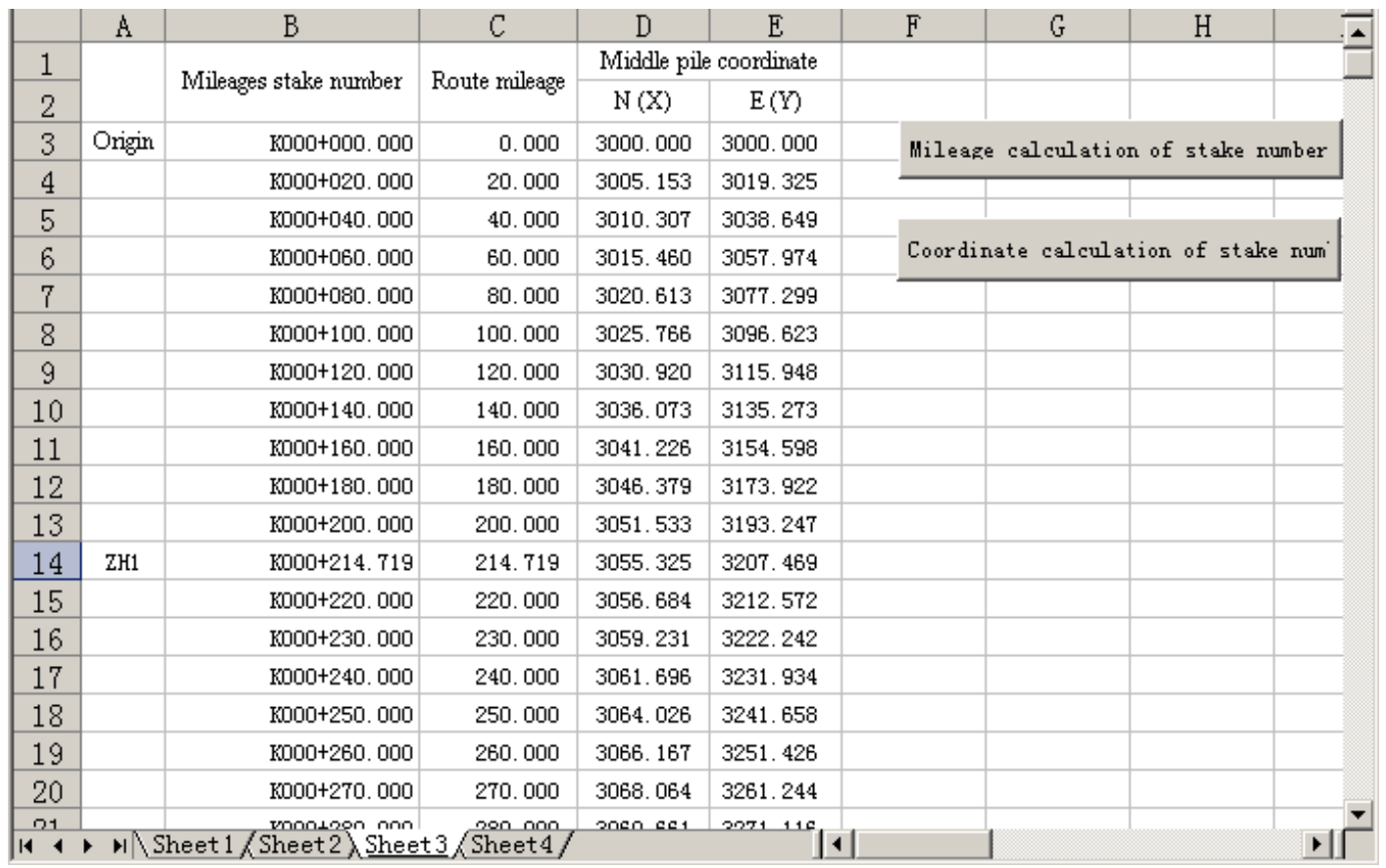

Fig.6. Pile mileage calculation and Pile coordinates calculation

Practices have proved that establishing calculation model according the measurement known data can effectively solve the problem and improve work efficiency in practical work. Due to the limitation of space, cannot give whole program source code, the rest of the program are the same with the design principle of part of given program.

Jilin province higher education teaching reform research subject: research on practice innovation ability training mode of civil engineering applied undergraduates.

\section{Reference}

[1]Guo-wen Che. An analogue orthotropic plate method to calculate load transverse distribution coefficients by using Excel 2011 International Conference on Electronic \& Mechanical Engineering and Information Technology (EMEIT 2011) 2011.08 pp3796-3799. 
[2]Guo-wen Che. Pearson-III frequency curve plotting in Excel table Applied Mechanics and Materials Vols. 556-562 (2014) pp 5829-5834.

[3]Nie Rang. Total station and high grade highway surveying[M]. People traffic press, 2005.

[4] Zhang Yu-hua. road surveying and design[M]. People traffic press, 2005.

[5] Xu Ya-ya, Luo Ying. Surveying[M]. People traffic press, 2003.

[6] Luo Gang-jun. Excel VBA programming self-study bible[M]. Electronic industry press,2014.

[7] John Walkenbach. Chinese version of Excel 2010 senior VBA programming[M]. Tsinghua university press, 2012. 